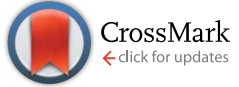

Cite this: RSC Adv., 2015, 5, 36036
Received 10th February 2015

Accepted 13th April 2015

DOI: $10.1039 / \mathrm{c} 5 \mathrm{ra02220 \textrm {g }}$

www.rsc.org/advances

\section{A single low-cost microfabrication approach for polymethylmethacrylate, polystyrene, polycarbonate and polysulfone based microdevices}

\author{
Jingyun Jiang, ${ }^{a}$ Jinsheng Zhan, ${ }^{a}$ Wanqing Yue, ${ }^{c}$ Mengsu Yang, ${ }^{c}$ Changqing $Y i^{* b}$ \\ and Cheuk-Wing Li ${ }^{\star a}$
}

\begin{abstract}
Although polydimethylsiloxane (PDMS) is the popular choice for prototyping microdevices, it suffers from non-specific surface adsorption, small molecule partitioning and drying issues. This paper presents a single microfabrication approach to prepare different thermoplastics that improved the issues inherent to PDMS microdevices. This approach involved transferring microfluidic features from a low-cost printed circuit board (PCB) by hot embossing followed by chloroform vapour assisted bonding. The micro features on the PCB master were comprised of copper metal which maintained structural integrity under pressure and high temperature, making this single microfabrication approach compatible with polycarbonate (PC), polymethylmethacrylate (PMMA), polystyrene (PS) and polysulfone (PSU) thermoplastics. We have modified the vaporized solvent bonding method so that lower temperature and pressure were sufficient for device bonding while the risk of microchannel deformation and clogging was minimized. Various fluorescent dyes and nanoparticles were used to demonstrate the better surface adsorption and partition properties of thermoplastics when compared with the PDMS counterpart. In addition, drying issues were markedly improved in the thermoplastic microdevices. This microfabrication method provided a single and low-cost approach to prepare various thermoplastic microdevices and the entire process (from PCB master to molding and bonding) was completed within 2 hours.
\end{abstract}

\section{Introduction}

Early microfluidic devices were made of either glass ${ }^{1}$ or silicon ${ }^{2}$ that involved expensive and complicated fabrication procedures. In response to the increasing demands for low-cost rapid microdevice prototyping, polymers have become a class of promising materials in microfabrication. Among all, elastomeric polydimethylsiloxane (PDMS) is the most popular choice for microdevices because the ease of fabrication by soft lithography ${ }^{3}$ and sealing by conformal contact. While gas permeability in PDMS is convenient for cell-culture applications, its porous nature have also attributed to the partition of organic solvents and small molecules, as demonstrated by fluorophores such as Nile red, ${ }^{4}$ quinine, ${ }^{4}$ Rhodamine $\mathrm{B}^{5}$ and drying issues ${ }^{6}$ due to water evaporation. Moreover, the native hydrophobic surface of PDMS causes strong non-specific adsorption of biological molecules like proteins. ${ }^{7}$ Since PDMS is not the optimal

${ }^{a}$ State Key Laboratory of Quality Research in Chinese Medicine, Institute of Chinese Medical Sciences, University of Macau, Macau SAR, China.E-mail: cheukwli@umac. mo; Fax: +8532884 1358; Tel: +853 88228513

${ }^{b}$ Key Laboratory of Sensing Technology and Biomedical Instruments, School of Engineering, Sun Yat-Sen University, Guangzhou, Guangdong Province, China

${ }^{c}$ Key Laboratory of Biochip Technology, Biotech and Health Centre, Shenzhen Research Institutes of City University of Hong Kong, Shenzhen, China choice for quantitative analysis in many circumstances, ${ }^{4,5}$ the need for low-cost microfabrication of a diversity of different polymers becomes increasingly important. Many thermoplastic polymers offer similar advantages like disposability, biocompatibility with acceptable optical transparency when comparing with PDMS $^{8}$ but their unique chemical and physical properties make them suitable for a particular type of microfluidic applications. ${ }^{\mathbf{9 - 1 1}}$

Various techniques are available for molding thermoplastic device, includes hot embossing, ${ }^{12}$ solvent imprinting, ${ }^{13}$ injection molding ${ }^{\mathbf{1 4}}$ and laser ablation. ${ }^{15}$ Among these techniques, hot embossing is widely employed because it is cost effective, easy to operate and suitable for the replication of various microstructures. However, the major challenge of hot embossing is the preparation of a master that resists high temperature and pressure. Masters have been prepared by nickel, ${ }^{\mathbf{8}}$ silicon, ${ }^{\mathbf{1 6}}$ SU8 (ref. 17) and plastic material. ${ }^{18}$ Silicon and SU8 masters have limited reusability due to cracking and distortion under high temperature and pressure. ${ }^{\mathbf{1 6 , 1 8 , 1 9}}$ Nickel master is very durable but expensive to prepare by micromachining. ${ }^{8}$ Plastic master often requires the preparation of a primary SU8 master $^{18}$ thus the fabrication process is indirect.

Effective bonding techniques are of equal importance in the fabrication of thermoplastics microdevices. Adhesive, ${ }^{20-22}$ thermal, ${ }^{9,23-30}$ solvent, ${ }^{18}$ microwave, ${ }^{31}$ ultrasonic $^{32}$ and surface 
modification bonding ${ }^{.33}$ methods have been reported but thermal bonding is the most commonly used bonding method. However, high temperature and pressure involved in thermal bonding may lead to channel deformation. ${ }^{34}$ Another popular choice for the bonding of thermoplastic device is solvent bonding. While solvent can be introduced on the substrate through sprinkling, ${ }^{35}$ capillary action ${ }^{36}$ and solvent bath dipping, ${ }^{18}$ it is difficult to avoid excessive solvent absorption that leads to collapse of microchannels. To tackle this issue, sacrificial techniques have been introduced by filling up the microchannels with ice ${ }^{34}$ or wax. ${ }^{37}$ Nevertheless, these bonding procedures require subsequent removal of residual sacrificial layer, which can be difficult for complex designs and inevitably complicated the fabrication process. Vaporized solvent bonding allows a controllable dose of solvent deposition so as to prevent excessive solvent absorption. A majority of vaporized solvent bonding methods are demonstrated by cyclic olefin copolymers ${ }^{38-43}$ that can be handled at room temperature.

In this paper, we described a single approach to prepare four different thermoplastic based microdevices: PMMA, PC, PS and PSU were selected due to their differences in glass transition temperatures as well as their suitability for different reported applications: PMMA provides better electrophoretic performance, ${ }^{9}$ PC is a good choice for PCR analysis due to its high glass transition temperature and low thermal conductivity. ${ }^{9}$ PS is a standard material for cell culture ${ }^{\mathbf{1 0}}$ and PSU exhibits outstanding hemocompatibility with blood. ${ }^{11}$ Printed circuit board (PCB) master can be used directly for hot embossing and it is durable after a hundred times of repeatedly embossment with negligible deformation of microstructures. We have finetuned the vaporized solvent bonding technique so that a lower temperature and pressure were applied for device bonding in order to minimize the risk of channel collapse/ deformation.

\section{Experimental section}

\section{Materials and reagents}

Several transparent thermoplastic materials, polymethylmethacrylate (PMMA), polystyrene (PS), polycarbonate (PC) and polysulfone (PSU), were purchased from ShengHua Plastic Corp. (Dongguan, China). ACS grade chloroform (IL, USA) was used for vaporized solvent bonding. 7-Amino-4-methylcoumarin (AMC) and fluorescein (FLUO) were purchased from Sigma (St. Louis, MO, USA). Rhodamine B (RhoB) was purchased from ACROS Organics. $200 \mu \mathrm{M}$ AMC, $10 \mu \mathrm{M}$ FLUO, $20 \mu \mathrm{M}$ RhoB and a solution of $2 \%$ fluorescent carboxylatemodified polystyrene microspheres (30 nm, L5155, Sigma St. Louis, MO, USA) were prepared in Hank's balanced salt solution (HBSS, Gibco, CarIsbad, CA, USA). For droplet formation, microfluidic channels were pre-treated with Aquapel (PPG Industries) and Fluorinert FC-40 (Sigma St. Louis, MO, USA) was used as oil phase with $2 \%(\mathrm{w} / \mathrm{w})$ surfactant (a poly(ethyleneglycol)di-krytox-FSH amide from RainDance Technologies, Lexington, MA).

\section{Instrumentation and data acquisition}

Hot embossing and vaporized solvent bonding were performed on a hot compressor (TM-101F, XinTaiming, China). To evaluate the dimension loss ratio of proposed fabrication method, the top view and cross-sectional images were taken from an inverse microscope (IX73, Olympus, Japan) equipped with a CCD camera (DP72, Olympus, Japan). Fluorescent images for partition/adsorption experiments were captured by an inverted fluorescent microscope with a Spinning Disk Confocal Scanner (IX81 DSU, Olympus, Japan). Droplet formation experiments were performed with a pair of syringe pumps (KDS270P, KD Scientific, USA). SEM images of PCB master were taken by JSM6010LA (JEOL, Japan). Talysurf CLI 1000 surface profiling system was used to obtain surface profile of new and 100-times used PCB masters.

\section{Fabrication of thermoplastic microfluidic devices}

Molding. Printed circuit board (PCB) masters (Kinsten, Chiefskill, Taiwan) were served as the embossing master for polymeric substrates with detailed procedure of master preparation being reported elsewhere. ${ }^{\mathbf{4 4 , 4 5}}$ Briefly, a photomask pattern was transferred to the photoresist of the PCB by UV irradiation. The exposed photoresist was developed and the unprotected copper layer was etched followed by removal of remaining photoresist to obtain a PCB master. A schematic of hot embossing procedure ${ }^{8}$ was shown in Fig. 1a. Prior to embossing, the polymeric sheets with a thickness of $1 \mathrm{~mm}$ were cut into a size of $3.5 \mathrm{~cm} \times 6.5 \mathrm{~cm}$. A substrate was sandwiched between the glass slide and PCB master, pressed and heated to form a mold. Once the plastics had been successfully embossed, the substrate was drilled with circular holes for solution inlets.

Bonding. The setup for vaporized solvent bonding was adopted from previous reports ${ }^{46}$ and illustrated in Fig. 1b. Chloroform solution was added in a $75 \mathrm{~mm}$ diameter glass Petri dish that was then sealed at room temperature for a period of time $\left(\mathrm{CHCl}_{3}\right.$ saturation time). Then, the seal of the Petri dish was removed and a glass cover holding the embossed substrate was used to close up the Petri dish. After a prescribed amount of time, the vapour treated substrate was brought into contact with another flat substrate of the same material. These two pieces of substrates were placed on soft support to ensure even pressure distribution and protected by a microscopic glass slide when

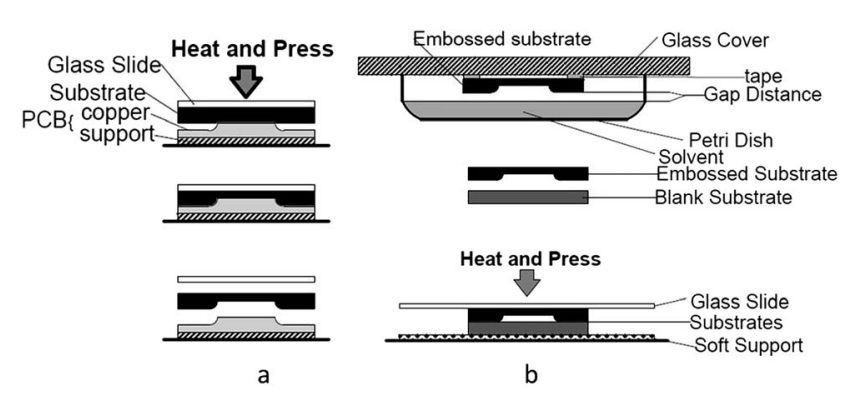

Fig. 1 Schematic diagram for device molding (a) and bonding (b). 
they were subjected to the final step of thermal bonding with pressure and temperature parameters tabulated in Table 2.

\section{Fabrication of PDMS microfluidic devices}

Poly(dimethylsiloxane) (PDMS) (RTV 615, GE Silicones, Waterford, NY, USA) was prepared by mixing the silicone elastomer with curing agent at weight ratio of $10: 1$. Degassed PDMS was poured over the PCB master and then cured at $80^{\circ} \mathrm{C}$ for $2 \mathrm{~h}$. The cured PDMS was peeled off from master. With access holes punched, the replica was sealed by dipping-attaching method. ${ }^{47}$

\section{Assessment of species adsorption and partition}

Microchannels were initially filled with buffer solution to obtain background fluorescent images. Then, images were captured every 20 second by flooding the channel with dye solution at flow rate of $325 \mu \mathrm{L} \mathrm{h}^{-1}$ for 15 min followed by washing with buffer at the same flow rate for another $20 \mathrm{~min}$. Backgroundcorrected fluorescence intensity profiles were obtained by ImageJ. Except for PDMS microdevices, epoxy adhesive was used to fix Teflon tubing inserted into the access ports of thermoplastics to ensure no solution leaking during the experiments.

\section{Droplet formation}

On-chip droplet formation was performed by using FC-40 fluorocarbon oil containing $2 \mathrm{wt} \%$ of PEG-based surfactant as the continuous phase and deionized water was chosen as the dispersed phase. Before conducting an experiment, microchannels were coated with Aquapel for at least $1 \mathrm{~min}$ followed by drying with air. Then, two immiscible phases were introduced into the device by two separated syringe pumps. The flow rates of oil phase and water phase were $30 \mu \mathrm{L} \mathrm{h}^{-1}$ and 10 respectively.

\section{Result and discussion}

Hot embossing is an effective way to produce microstructures by directly stamping patterns into heated thermoplastic materials but a robust master that withstands high mechanical stress and temperature is a prerequisite. PCB masters are inexpensive, easily attainable, with robust copper based features that retained good condition after repeatedly molding for a hundred times. In our experiment, PCB masters were used to stamp on a number of thermoplastics with glass transition temperature $\left(T_{\mathrm{g}}\right)$ ranging from $105{ }^{\circ} \mathrm{C}$ to $190{ }^{\circ} \mathrm{C}$. Fig. 2a showed an SEM image of a 100-times used PCB master. Based on the profilometry results, the height of microchannels were similar in the new and 100-times used masters while the mean roughness $\left(R_{\mathrm{a}}\right)$ values of new and used PCB masters were $0.56 \pm 0.13 \mu \mathrm{m}$ and $0.67 \pm 0.22 \mu \mathrm{m}$ respectively, suggested high reusability of the PCB master when molding against rigid thermoplastics.

Parameters used for the embossing and bonding different thermoplastics were tabulated in Tables 1 and 2 respectively. In molding processes, embossing temperature was set to be $20^{\circ} \mathrm{C}$ above the glass transition temperature of the corresponding thermoplastic material, except for PSU where a 2-step
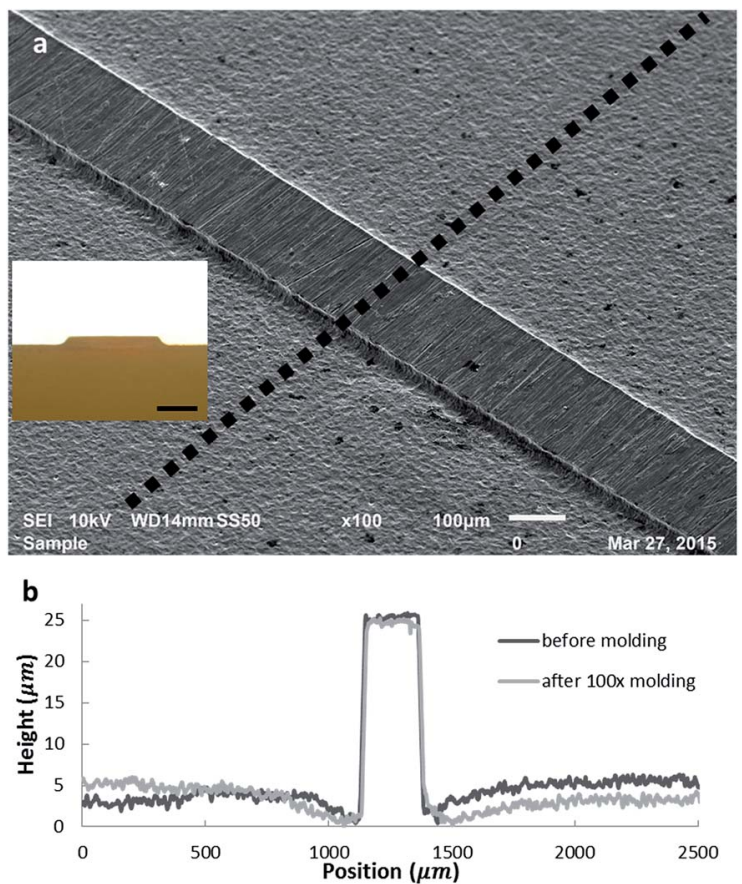

Fig. 2 (a) Tilted SEM image of a PCB master repeatedly used for 100 times with inset showing the master cross-section. Scale bar is 100 $\mu \mathrm{m}$. (b) Height measurement of new and 100-times used PCB masters were taken by a profilometer along the black dotted line depicted in (a).

Table 1 Conditions for hot embossing against PCB masters

\begin{tabular}{|c|c|c|c|c|}
\hline \multirow[b]{2}{*}{ Substrate } & \multirow[b]{2}{*}{$T_{\mathrm{g}}$} & \multicolumn{3}{|l|}{ Hot embossing } \\
\hline & & Temp & Time & Pressure \\
\hline oint & & & & \\
\hline PS & $105^{\circ} \mathrm{C}$ & $125^{\circ} \mathrm{C}$ & $10 \mathrm{~min}$ & 92 psi \\
\hline PMMA & $120^{\circ} \mathrm{C}$ & $140^{\circ} \mathrm{C}$ & $5 \min$ & 92 psi \\
\hline $\mathrm{PC}$ & $150^{\circ} \mathrm{C}$ & $170{ }^{\circ} \mathrm{C}$ & $15 \mathrm{~min}$ & 92 psi \\
\hline PSU & $190^{\circ} \mathrm{C}$ & $\begin{array}{l}\text { Step } 1220{ }^{\circ} \mathrm{C} \\
\text { Step } 2220 \rightarrow 190{ }^{\circ} \mathrm{C}\end{array}$ & $\begin{array}{l}\text { Step } 18 \mathrm{~min} \\
\text { Step } 25 \mathrm{~min}\end{array}$ & $92 \mathrm{psi}$ \\
\hline
\end{tabular}

embossing temperature was used to ensure successful molding due to the minor difference between the glass transition and melting temperature of PSU.

Conventional vaporized solvent bonding methods consider only the solvent vapour time, i.e., the duration that a substrate is treated with the vapour of bonding solvent. Here, we further defined two waiting period to increase bonding reproducibility (Table 2). (i) Before each bonding process, chloroform was sealed in the Petri dish for a certain period of time, i.e. the " $\mathrm{CHCl}_{3}$ saturation time" before incubating the embossed substrate (ii) after the vaporized substrate was gently brought into contact with a blank substrate prior to thermal bonding, another waiting period was introduced, i.e., the " evaporation time".

The distance between chloroform liquid surface and embossed substrate was critical to bonding performance and reported as the gap distance in Table 2. The $\mathrm{CHCl}_{3}$ saturation 
Table 2 Conditions for vaporized solvent bonding

\begin{tabular}{|c|c|c|c|c|c|c|c|}
\hline \multirow[b]{2}{*}{ Substrate } & \multirow[b]{2}{*}{$\mathrm{CHCl}_{3}$ saturation time } & \multirow[b]{2}{*}{ Gap distance } & \multirow[b]{2}{*}{$\mathrm{CHCl}_{3}$ vapour time } & \multirow[b]{2}{*}{ Evaporation time } & \multicolumn{3}{|c|}{ Bonding parameters } \\
\hline & & & & & Temp & Time & Pressure \\
\hline PS & $4 \min$ & $12.5 \mathrm{~mm}$ & $30 \mathrm{~s}$ & $2 \min$ & $85^{\circ} \mathrm{C}$ & $30 \mathrm{~s}$ & $70 \mathrm{psi}$ \\
\hline PMMA & $10 \mathrm{~min}$ & $7.5 \mathrm{~mm}$ & $60 \mathrm{~s}$ & $5 \mathrm{~min}$ & $100^{\circ} \mathrm{C}$ & $45 \mathrm{~s}$ & $80 \mathrm{psi}$ \\
\hline PC & $10 \mathrm{~min}$ & $10.0 \mathrm{~mm}$ & $35 \mathrm{~s}$ & $3 \mathrm{~min}$ & $100{ }^{\circ} \mathrm{C}$ & $60 \mathrm{~s}$ & $70 \mathrm{psi}$ \\
\hline PSU & $15 \mathrm{~min}$ & $7.5 \mathrm{~mm}$ & $60 \mathrm{~s}$ & $2 \min$ & $140^{\circ} \mathrm{C}$ & $60 \mathrm{~s}$ & $80 \mathrm{psi}$ \\
\hline
\end{tabular}

time and gap distance determined the vapour environment inside the Petri dish before substrate exposure. While increasing the saturation time and decreasing the gap distance facilitated bonding, too much vapour being deposited on the substrate would lead to clogged microchannels and increased the overall fabrication time. The $\mathrm{CHCl}_{3}$ vapour time was the duration when substrate was exposed to preconditioned vapour environment. After vaporized substrate was brought into contact with another piece of substrate, the sandwich was transferred onto the stage of hot compressor for thermal bonding. We found that another source of bonding irreproducibility was originated from the duration of this handling time so the "evaporation time" was defined to ensure a consistent period of time was given before embossing. If evaporation time exceeded the designated period, the escape of chloroform vapour from the sandwich would lead to incomplete bonding. During the fine-tuning of parameters in Table 2, when some vaporized conditions leaded to too short a evaporation time, $\mathrm{CHCl}_{3}$ saturation time and gap distanced were adjusted accordingly in order to increase vapour exposure on substrates.

The final bonding step, vaporized solvent bonding, was dependent to temperature, pressure and the duration of bonding. By comparing our modified vaporized solvent bonding process with other thermal bonding processes found in literatures (Table 3), our method required a significantly shorter compression time of about 1 minute and the bonding temperature used were below the glass transition temperatures of corresponding substrates to minimize channel deformation.

Table 3 Literature summary of thermal bonding conditions

\begin{tabular}{|c|c|c|c|c|}
\hline \multirow[b]{2}{*}{ Author } & \multirow[b]{2}{*}{ Substrate } & \multicolumn{3}{|c|}{ Bonding parameters } \\
\hline & & Temp & Time & Pressure \\
\hline Chen $^{9}$ & $\mathrm{PC}$ & $150{ }^{\circ} \mathrm{C}$ & $20 \mathrm{~min}$ & (No pressure) \\
\hline $\operatorname{Sun}^{28}$ & PMMA & $165^{\circ} \mathrm{C}$ & $30 \mathrm{~min}$ & $20 \mathrm{kPa}(3 \mathrm{psi})$ \\
\hline $\operatorname{Tan}^{29}$ & PMMA & $160{ }^{\circ} \mathrm{C}$ & $30 \mathrm{~min}$ & (No pressure) \\
\hline Locascio $^{26}$ & PS & $105^{\circ} \mathrm{C}$ & $10 \mathrm{~min}$ & (No pressure) \\
\hline \multirow[t]{4}{*}{ Shadpour $^{27}$} & PSU & $185^{\circ} \mathrm{C}$ & $30 \mathrm{~min}$ & (No pressure) \\
\hline & PS & $110^{\circ} \mathrm{C}$ & $15 \min$ & (No pressure) \\
\hline & $\mathrm{PC}$ & $150{ }^{\circ} \mathrm{C}$ & $20 \min$ & (No pressure) \\
\hline & PMMA & $107^{\circ} \mathrm{C}$ & $20 \mathrm{~min}$ & (No pressure) \\
\hline Yang $^{30}$ & PC & $133{ }^{\circ} \mathrm{C}$ & $2 \mathrm{~h}$ & $1400 \mathrm{psi}$ \\
\hline $\mathrm{Li}^{24}$ & PC & $138{ }^{\circ} \mathrm{C}$ & $10 \mathrm{~min}$ & 900 psi \\
\hline $\mathrm{Buch}^{23}$ & $\mathrm{PC}$ & $140{ }^{\circ} \mathrm{C}$ & $10 \mathrm{~min}$ & $900 \mathrm{psi}$ \\
\hline $\mathrm{Liu}^{25}$ & PMMA & $85^{\circ} \mathrm{C}$ & Step 1: $5 \mathrm{~min}$ & Step 1: 44 psi \\
\hline
\end{tabular}

It is also worth noting that a majority of reports using conventional vaporized solvent bonding have only demonstrated their feasibility by one substrate material (mainly cyclic olefins copolymer, Table 4). Here, we have successfully produced microchips based on PC, PMMA, PS and PSU materials by using relatively lower pressure and shorter bonding time, which was helpful to minimize channel deformation/collapse. The entire fabrication process (from PCB preparation, molding to bonding) could be completed within 2 hours, which is suitable for the purpose of rapid prototyping microdevices. Although vaporized solvent bonding involved only minute amount of $\mathrm{CHCl}_{3}$ should have permeated after the final bonding step with elevated temperature ${ }^{18}$ copious rinsing of mold plastics is recommended to remove any residual chloroform which may do harm to cells or interfere with biological experiments.

The top view and cross-section of the bonded chips were illustrated in Fig. 3. The cross sections of the microchannels were obtained by mechanical cutting. Fig. 3a and c compared the top-view intersecting channel of different thermoplastics with PDMS and all these moulds were prepared from an identical PCB master. Among all thermoplastics, PMMA demonstrated the best fidelity in reproducing the feature from its master, as shown by the more well-defined corners of the intersecting channel. Details about the widths and depths of microchannels after embossing and bonding processes were plotted in Fig. 4. Fig. 3d showed the cross section of the thermoplastics and channel shapes were resemblance to the PDMS counterpart (Fig. 3b). Moreover, in each of these cross sections image, the two layers of substrates were completely merged together, an indicative of good bonding quality.

A quantitative comparison of channel dimension before and after bonding was plotted in Fig. 4. Each column in the plot represented an averaged result of 5 replicas. The small standard deviations in each thermoplastics suggested the high reproducibility in the embossing and bonding processes. PDMS replicas were used as a reference for the actual dimension of the PCB master. Compared with PDMS, the embossed channel depth and width of PMMA and PS were essentially the same while PC and PSU were slightly decreased. In addition, the dimension loss percentage (the channel dimension difference between embossed substrate and bonded chip over the dimension of embossed substrate) were showed in dotted lines. It was clear that PMMA demonstrated the smallest deviation in channel dimensions among all thermoplastics. The average depth of the bonded channels was $24.5 \mu \mathrm{m}$ with the loss percentage of $4.38 \%$. Similarly, the average width measured at 
Table 4 Literature summary of vaporized solvent bonding conditions

Bonding parameters

\begin{tabular}{|c|c|c|c|c|c|c|}
\hline Author & Substrate & Solvent & Vapour time & Temp & Time & Pressure \\
\hline \multirow[t]{2}{*}{ Ogilvie $^{46}$} & PMMA & Chloroform & $4 \min \left(25^{\circ} \mathrm{C}\right)$ & $65^{\circ} \mathrm{C}$ & $20 \mathrm{~min}$ & 203 psi $\left(140 \mathrm{~N} \mathrm{~cm}^{-1}\right)$ \\
\hline & $\mathrm{COC}$ & Cyclohexane & $4 \min \left(25^{\circ} \mathrm{C}\right)$ & $65^{\circ} \mathrm{C}$ & $20 \min$ & 203 psi $\left(140 \mathrm{~N} \mathrm{~cm}^{-1}\right)$ \\
\hline Rahmanian $^{38}$ & $\mathrm{COC}$ & Cyclohexane & $15 \min \left(30^{\circ} \mathrm{C}\right)$ & RT & (Not mentioned) & 500 psi \\
\hline Chen $^{39}$ & $\mathrm{COP}$ & Cyclohexane & $7.5 \min \left(30^{\circ} \mathrm{C}\right)$ & RT & $1 \mathrm{~min}$ & 2000 psi (13.79 MPa) \\
\hline Chen $^{40}$ & $\mathrm{COC}$ & Cyclohexane & $7 \min \left(30^{\circ} \mathrm{C}\right)$ & RT & $10 \mathrm{~min}$ & 500 psi (3.45 MPa) \\
\hline Liu $^{41}$ & $\mathrm{COC}$ & Methylcyclohexane & $1 \mathrm{~min}, \mathrm{RT}$ & $40{ }^{\circ} \mathrm{C}$ & $10 \mathrm{~min}$ & 200 psi \\
\hline $\mathrm{Ro}^{42}$ & $\mathrm{COC}$ & Methylcyclohexane & $3 \mathrm{~min}, \mathrm{RT}$ & $70{ }^{\circ} \mathrm{C}$ & $10 \mathrm{~min}$ & 100 psi \\
\hline Sauer $^{43}$ & $\mathrm{COC}$ & Toluene & $1.5 \mathrm{~h}, \mathrm{RT}$ & $133{ }^{\circ} \mathrm{C}$ & $2 \min$ & $1125 \mathrm{psi}$ \\
\hline Ogonczyk $^{48}$ & $\mathrm{PC}$ & Dichloromethane & $30 \min \left(25^{\circ} \mathrm{C}\right)$ & $125-135^{\circ} \mathrm{C}$ & $30 \mathrm{~min}$ & $58 \mathrm{psi}(0.4 \mathrm{MPa})$ \\
\hline
\end{tabular}

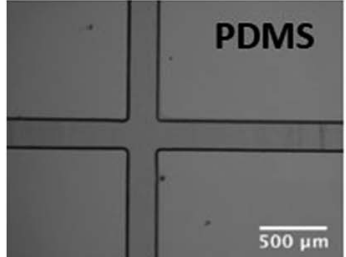

a
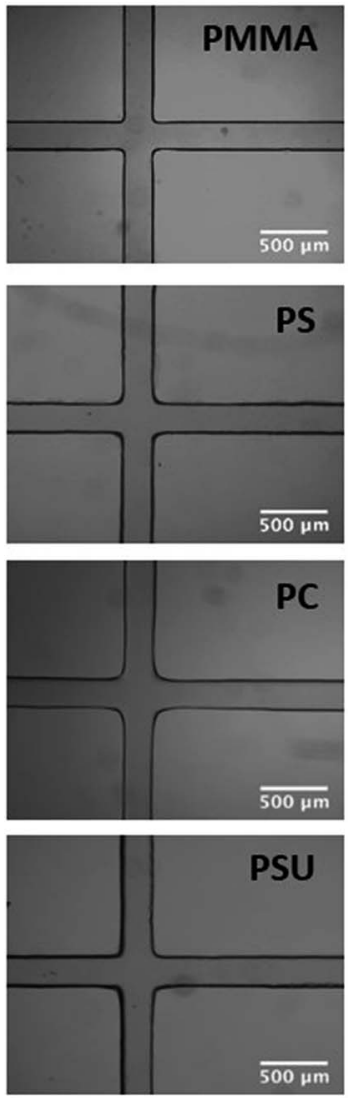

C

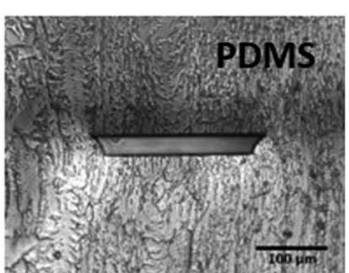

b
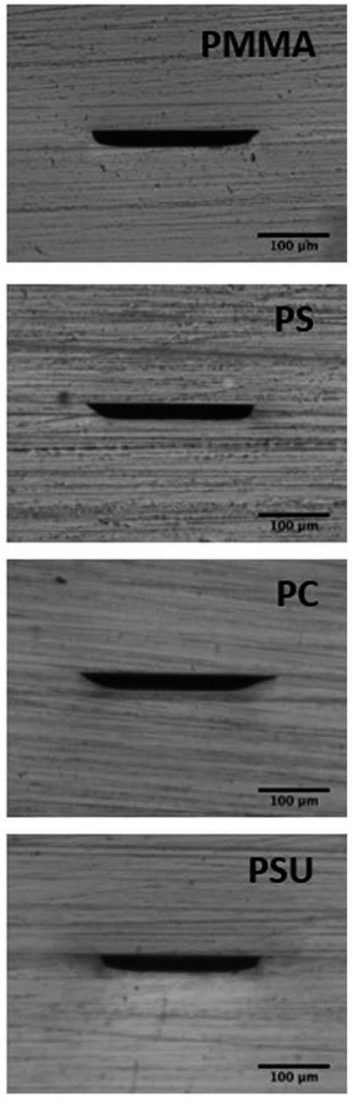

d
Fig. 3 Top-view ( $a$ and $c$ ) and cross-section ( $b$ and $d$ ) images of microdevices based on different substrates. The dimension of PDMS based microdevices were used as reference to the actual dimension on PCB board. the bottom of inverted trapezoidal shaped cross section (Fig. 3d) was $198 \mu \mathrm{m}$ and the loss percentage was less than $3 \%$. However, PS and PSU were subjected to a relatively greater loss in dimension after the bonding process. Overall, the bonding quality was satisfactory with dimension loss percentage below $10 \%$ among all thermoplastics, which should be acceptable for many microfluidic applications.

In order to evaluate the quality and usability of these thermoplastic chips, a series of adsorption and partition tests were performed. The prerequisite of these tests was leak-free bonding among the thermoplastic devices. Except for PDMS microdevices, epoxy adhesive was used to fix Teflon tubing inserted into the access ports of thermoplastics to ensure no solution leaking occurred at the reservoirs throughout the experiments. All types of thermoplastic chips have passed this leakage test by withstanding a flow speed of $325 \mu \mathrm{L} \mathrm{h}{ }^{-1}$ for at least half an hour.

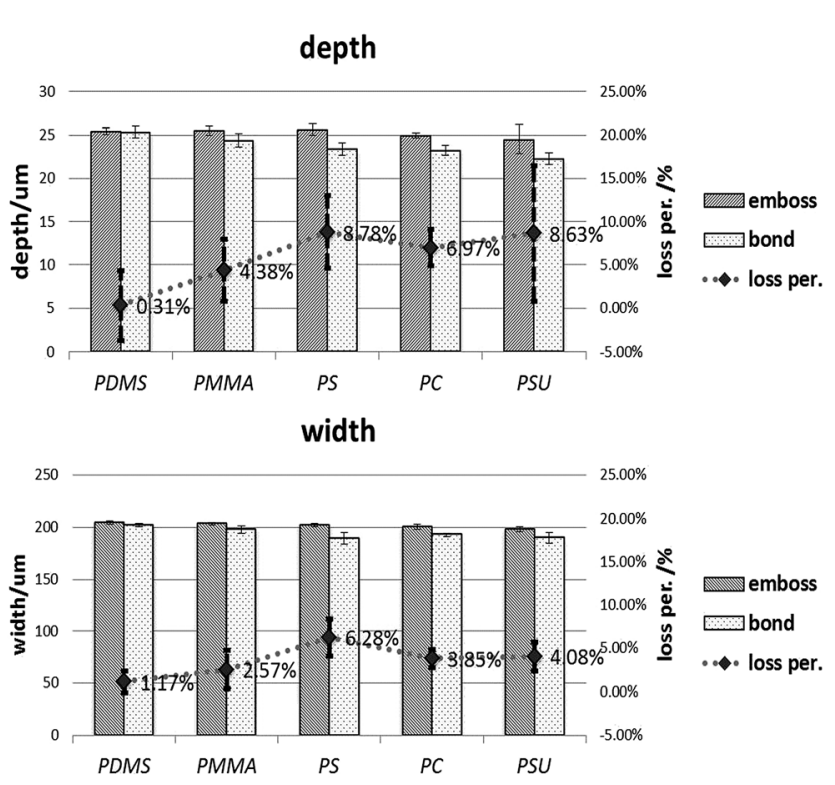

Fig. 4 Depth and width of microchannels after molding (grey bars) and bonding (white bars) were plotted with their dimension loss percentage (dotted lines). For each bar, results were obtained from 5 individual replicas molded against an identical PCB master. 
High adsorption (on surface) and partition (into bulk) of certain small molecules and proteins are notorious to PDMS based microfluidic devices due to its porous and hydrophobic nature, ${ }^{5,7}$ which compromised the accuracy of quantitative analysis in certain assays. ${ }^{4}$ Thus, adsorption and partition of dye and particles were examined in thermoplastic microchips and PDMS microchips for comparison. We have selected carboxylate-modified, fluorescent microspheres and three fluorescent dyes excitable by commonly used filter sets (DAPI, FITC and TRITC) in fluorescence microscopy. Fig. 5 showed a PDMS based microchannel filled with Rhodamine B (RhoB) dye followed by buffer rinsing, and a significant residual fluorescent signal could be detected. The percentage of species adsorption was determined by the normalization of background subtracted residual fluorescence signal by the background subtracted dye filled signal. In addition to surface adsorption, some species tended to enter the PDMS porous matrix. Such species partition was quantified by the evaluation of the partition distance as showed in Fig. 5 , i.e., the distance where fluorophores travelled into the bulk of the substrate. Partition rate $\left(\mu \mathrm{m} \mathrm{min}^{-1}\right)$ of each species was calculated by the partition distance over the duration of dye fill time. The quantitative analysis of species adsorption and partition in varies thermoplastics with PDMS elastomer were shown in Fig. 6. Referring to the left $y$-axis that represented the percentage adsorption of species on microchannel surface, all 4 thermoplastics and PDMS exhibited considerable surface adsorption ( $20 \%$ or above) to the $30 \mathrm{~nm}$, carboxylate-modified polystyrene (L5155) after microchannels being continuously rinsed with buffer at $325 \mu \mathrm{L} \mathrm{h}^{-1}$ for $20 \mathrm{~min}$. While Rhodamine B (RhoB) is well-known to be severely adsorbed on PDMS surface, ${ }^{5}$ this commonly used dye was compatible with other thermoplastics and worked particularly well with PMMA. 7-Amino-4-methyl-coumarin (AMC) and fluorescein (FLUO) were compatible with all thermoplastics and the elastomer while these dyes worked particularly well with PS with negligible adsorption. By referring the right $y$-axis that represented the rate of species entering the bulk of materials in

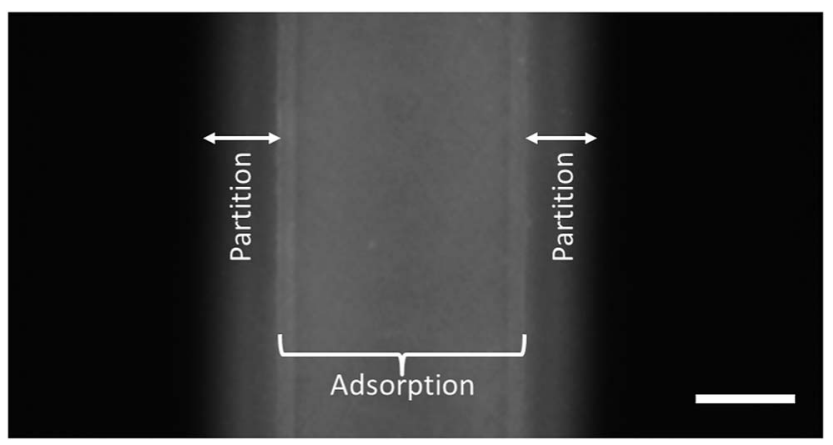

Fig. 5 Adsorption and partition of species in microchannel: fluorescence image of a microchannel (PDMS substrate was used as an example) was acquired after filling with RhoB for 15 min followed by buffer rinsing for $20 \mathrm{~min}$. The residual signal within the area of microchannel (confirmed by bright field image) was defined as dye adsorption while the distance per min of fluorescence signal detected outside the microchannel was defined as partition rate.

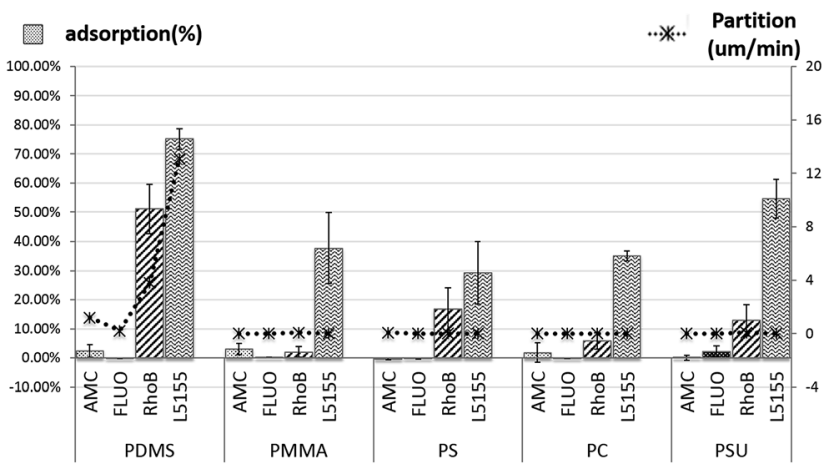

Fig. 6 Adsorption and partition of different fluorescent dyes and nanoparticles. Bar chart represented the species adsorption on the wall of microchannel (the left $y$-axis) while the dotted lines represented the partition rate of species into the bulk of polymers (the right $y$-axis).

Fig. 6, PDMS elastomer was clearly inferior to other thermoplastics and severely partitioned by $30 \mathrm{~nm}$ nanoparticles, RhoB and AMC. In contrast, all other thermoplastics exhibited negligible partition of the species used in this study. Accordingly, AMC, FLUO and RhoB, which worked in DAPI, FITC, TRITC filter sets respectively, have performed well by having low adsorption and partition in various thermoplastics and they were suitable candidates for on-chip quantitative analysis.

While the porous nature of PDMS attributed to the uptake of small molecules as shown in the partition of dye species, it also facilitated the diffusion of water vapour into the bulk polymer. Our second challenge to these thermoplastic devices was their ability to tackle the notorious drying issues associated with PDMS microdevices. ${ }^{6}$ We leverage the capability of microfluidic droplet formation to prepare well-defined water droplets followed by monitoring the change in droplet size during the course of on-chip incubation to study the extent of water drying among all substrates.

The microchip design layout was shown in Fig. 7. This widely adopted droplet formation design was comprised of two inlets,

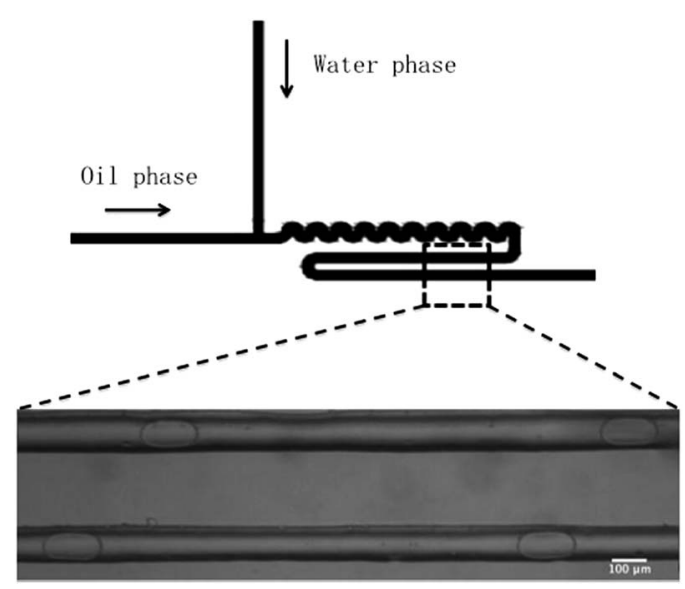

Fig. 7 Design layout of the droplet formation microdevice with inset showing the micrograph of droplets maintained steadily within a PCbased microchannel for incubation. 


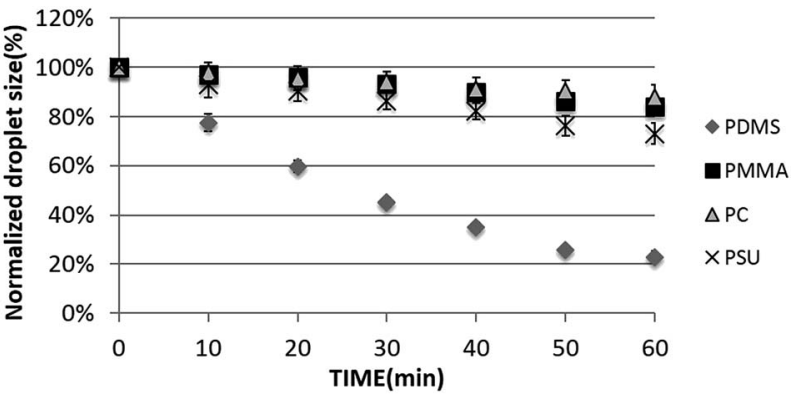

Fig. 8 Influence of substrate material on droplet size during 1 hour on-chip incubation at room temperature. The normalized droplet size was calculated by normalizing the residual droplet volume at a particular time point over the original droplet size at time zero.

one for dispersed phase (water) and the other for continuous phase (oil). The main channel dimensions were $20 \mu \mathrm{m}$ in depth and $90 \mu \mathrm{m}$ in width. After uniform droplet formation was achieved, the tubings of oil and water inlets were removed such that individual droplets were retained within the microchannel at even spacing as illustrated in the inset of Fig. 7. Fig. 8 showed the relationship between the normalized droplet size (by normalizing the residual droplet volume at a particular time point over the original droplet size at zero time point) and their incubation time inside the microchannels made of different substrates. Since PS was incompatible with Aquapel coating (severe cracking resulted once the chemical filled the microchannel), the substrate was excluded from this test. For the remaining candidates, images captured at 10 minutes intervals were used for the evaluation of droplet size change as a function of time.

The on-chip experiments for different substrates were performed side-by-side so variation in ambient temperature and humidity were negligible. During the entire one-hour observation period, the evenly spaced droplets remained static within the microchannels, however, $\sim 80 \%$ droplet size shrinkage was observed in PDMS based microdevices. PC has demonstrated the minimal size shrinkage by retaining almost $90 \%$ of its original droplet size. All the tested thermoplastics have performed significantly better than the PDMS elastomer in terms of maintaining droplet size and the results were consistent with their low species partition. The results also suggested it is straightforward to adopt thermoplastic microdevices to conduct droplet-based chemical reactions so as to minimize the impact of volume lost accompanying with PDMS substrates.

\section{Conclusion}

We have demonstrated a single, cost-effective microfabrication method that is suitable for thermoplastics with glass transition temperature lower than $190{ }^{\circ} \mathrm{C}$. Four different types of thermoplastic substrates, PS, PMMA, PC and PSU, have been employed for device fabrication and the entire fabrication process, from PCB master preparation, molding to device bonding, could be completed within 2 hours. We have evaluated the reusability of PCB master and the fidelity of channel dimension reproduced on thermoplastic microdevices. The copper based microstructures featured on the PCB master were durable enough for a hundred times of repeatedly embossing against rigid thermoplastics material. This method produced microchips with sufficient bonding strength to carry out experiments at a flow rate of $325 \mu \mathrm{L} \mathrm{h}^{-1}$ for at least half an hour without solution leaking. Among all tested thermoplastics, PMMA demonstrated the best fidelity in reproducing the features from its master so that smallest deviation in channel dimension was observed after molding and bonding. PS, a commonly used plastic material for disposable cell-culture consumables, may better serve for quantitative assays because of its negligible partition and adsorption properties. In addition, drying issues inherent in porous PDMS could be markedly improved by using thermoplastics materials, as shown in the droplet formation experiment where the volume of on-chip generated microdroplets was better maintained in all tested thermoplastics when compared with PDMS devices. This microfabrication method provided a single approach to prepare various thermoplastics microdevices which should fit the needs for experiments having different requirements to surface properties and tolerance to drying issues.

\section{Acknowledgements}

C.-W. Li would like to thank Professor Richard N. Zare for his guidance during Li's postdoctoral study in Stanford University. The work was supported by grants from the University of Macau (MYRG108(Y1-L2)-ICMS13-LCW, MYRG2014-00179-ICMS-QRCM), the National Natural Science Foundation of China (51102097), the Basic Research Program of Shenzhen Science and Technology Innovation Plan (JC201105201055A) and Guangzhou Science and Technology and Information Bureau (2013J2200053, 2014J4100108).

\section{Notes and references}

1 D. J. Harrison, A. Manz, Z. H. Fan, H. Ludi and H. M. Widmer, Anal. Chem., 1992, 64, 1926-1932.

2 A. Manz, J. C. Fettinger, E. Verpoorte, H. Ludi, H. M. Widmer and D. J. Harrison, TrAC, Trends Anal. Chem., 1991, 10, 144149.

3 H. Makamba, J. H. Kim, K. Lim, N. Park and J. H. Hahn, Electrophoresis, 2003, 24, 3607-3619.

4 M. W. Toepke and D. J. Beebe, Lab Chip, 2006, 6, 1484-1486.

5 H. Sasaki, H. Onoe, T. Osaki, R. Kawano and S. Takeuchi, Sens. Actuators, B, 2010, 150, 478-482.

6 Y. Zhang, M. Ishida, Y. Kazoe, Y. Sato and N. Miki, IEEJ Trans. Electr. Electron. Eng., 2009, 4, 442-449.

7 B. Huang, H. Wu, S. Kim and R. N. Zare, Lab Chip, 2005, 5, 1005-1007.

8 H. Becker and U. Heim, Sens. Actuators, A, 2000, 83, 130-135. 9 J. F. Chen, M. Wabuyele, H. W. Chen, D. Patterson, M. Hupert, H. Shadpour, D. Nikitopoulos and S. A. Soper, Anal. Chem., 2005, 77, 658-666.

10 H. W. Li, Y. Q. Fan, R. Kodzius and I. G. Foulds, Microsyst. Technol., 2012, 18, 373-379. 
11 M. C. Yang and W. C. Lin, J. Polym. Res., 2002, 9, 61-67.

12 L. Martynova, L. E. Locascio, M. Gaitan, G. W. Kramer, R. G. Christensen and W. A. MacCrehan, Anal. Chem., 1997, 69, 4783-4789.

13 X. Sun, B. A. Peeni, W. Yang, H. A. Becerril and A. T. Woolley, J. Chromatogr. A, 2007, 1162, 162-166.

14 R. M. McCormick, R. J. Nelson, M. G. AlonsoAmigo, J. Benvegnu and H. H. Hooper, Anal. Chem., 1997, 69, 2626-2630.

15 D. L. Pugmire, E. A. Waddell, R. Haasch, M. J. Tarlov and E. Locascio, Anal. Chem., 2002, 74, 871-878.

16 M. B. Esch, S. Kapur, G. Irizarry and V. Genova, Lab Chip, 2003, 3, 121-127.

17 J. C. McDonald, D. C. Duffy, J. R. Anderson, D. T. Chiu, H. K. Wu, O. J. A. Schueller and G. M. Whitesides, Electrophoresis, 2000, 21, 27-40.

18 M. T. Koesdjojo, C. R. Koch and V. T. Remcho, Anal. Chem., 2009, 81, 1652-1659.

19 S. R. Nugen, P. J. Asiello and A. J. Baeumner, Microsyst. Technol., 2009, 15, 477-483.

20 F. Dang, S. Shinohara, O. Tabata, Y. Yamaoka, M. Kurokawa, Y. Shinohara, M. Ishikawa and Y. Baba, Lab Chip, 2005, 5, 472-478.

21 M. W. Boehm, S. Sarker and K. Koelling, Microfluid. Nanofluid., 2011, 10, 1175-1183.

22 M. W. Boehm and K. W. Koelling, Polym. Eng. Sci., 2012, 52, 1590-1599.

23 J. S. Buch, C. Kimball, F. Rosenberger, W. E. Highsmith, D. L. DeVoe and C. S. Lee, Anal. Chem., 2004, 76, 874-881.

24 Y. Li, J. S. Buch, F. Rosenberger, D. L. DeVoe and C. S. Lee, Anal. Chem., 2004, 76, 742-748.

25 J. Liu, S. Yang, C. S. Lee and D. L. DeVoe, Electrophoresis, 2008, 29, 2241-2250.

26 L. E. Locascio, C. E. Perso and C. S. Lee, J. Chromatogr. A, 1999, 857, 275-284.

27 H. Shadpour, H. Musyimi, J. Chen and S. A. Soper, J. Chromatogr. A, 2006, 1111, 238-251.

28 Y. Sun, Y. C. Kwok and N.-T. Nguyen, J. Micromech. Microeng., 2006, 16, 1681-1688.

29 H. Y. Tan, W. K. Loke, Y. T. Tan and N. T. Nguyen, Lab Chip, 2008, 8, 885-891.
30 J. Yang, Y. Liu, C. B. Rauch, R. L. Stevens, R. H. Liu, R. Lenigk and P. Grodzinski, Lab Chip, 2002, 2, 179-187.

31 A. A. Yussuf, I. Sbarski, J. P. Hayes, M. Solomon and N. Tran, J. Micromech. Microeng., 2005, 15, 1692-1699.

32 Z. B. Zhang, Y. Luo, X. D. Wang, Z. Q. Zhang and L. D. Wang, Int. Conf. Electron. Packag. Technol. High Density Packag., 13th, 2008, 1-2, 34-38, DOI: 10.1109/Fskd.2008.583.

33 M. J. Shenton, M. C. Lovell-Hoare and G. C. Stevens, J. Phys. D: Appl. Phys., 2001, 34, 2754-2760.

34 M. T. Koesdjojo, Y. H. Tennico, J. T. Rundel and V. T. Remcho, Sens. Actuators, B, 2008, 131, 692-697.

35 C. Lin, C. Chao and C. Lan, Sens. Actuators, B, 2007, 121, 698705.

36 J. J. Shah, J. Geist, L. E. Locascio, M. Gaitan, M. V. Rao and W. N. Vreeland, Anal. Chem., 2006, 78, 3348-3353.

37 R. T. Kelly, T. Pan and A. T. Woolley, Anal. Chem., 2005, 77, 3536-3541.

38 O. Rahmanian and D. L. DeVoe, Lab Chip, 2013, 13, 11021108.

39 C. F. Chen, J. Liu, C. C. Chang and D. L. DeVoe, Lab Chip, 2009, 9, 3511-3516.

40 C. F. Chen, J. Liu, L. P. Hromada, C. W. Tsao, C. C. Chang and D. L. DeVoe, Lab Chip, 2009, 9, 50-55.

41 J. Liu, K.-W. Ro, R. Nayak and D. R. Knapp, Int. J. Mass Spectrom., 2007, 259, 65-72.

42 K. W. Ro, J. Liu and D. R. Knapp, J. Chromatogr. A, 2006, 1111, 40-47.

43 A. F. Sauer-Budge, P. Mirer, A. Chatterjee, C. M. Klapperich, D. Chargin and A. Sharon, Lab Chip, 2009, 9, 2803-2810.

44 W. Yue, C. W. Li, T. Xu and M. Yang, Lab Chip, 2011, 11, 3352-3355.

45 C.-W. Li, C. N. Cheung, J. Yang, C. H. Tzang and M. Yang, Analyst, 2003, 128, 1137.

46 I. R. G. Ogilvie, V. J. Sieben, C. F. A. Floquet, R. Zmijan, M. C. Mowlem and H. Morgan, J. Micromech. Microeng., 2010, 20, 065016.

47 H. Wu, B. Huang and R. N. Zare, Lab Chip, 2005, 5, 13931398.

48 D. Ogonczyk, J. Wegrzyn, P. Jankowski, B. Dabrowski and P. Garstecki, Lab Chip, 2010, 10, 1324-1327. 